\title{
LYME DISEASE ASSOCIATED NEURORETINITIS - CASE REPORT
}

\author{
MElinda VANYA ${ }^{1 *}$, ImRe FeJES ${ }^{3}$, MARIA JAKO $^{1}$, AReta Tula ${ }^{2}$, \\ GABRIELLA TERHES $^{4}$, MARTA JANAKY ${ }^{3}$ and GYORGY BARTFAI ${ }^{1}$ \\ ${ }^{1}$ Department of Obstetrics and Gynaecology, Faculty of Medicine, \\ Albert Szent-Gyorgyi Clinical Centre, University of Szeged, Szeged, Hungary \\ ${ }^{2}$ Department of Obstetrics and Gynaecology, Riga Stradiņš University, Riga, Latvia \\ ${ }^{3}$ Department of Ophthalmology, Faculty of General Medicine, \\ Albert Szent-Gyorgyi Clinical Centre, University of Szeged, Szeged, Hungary \\ ${ }^{4}$ Department of Clinical Microbiology, Faculty of General Medicine, \\ Albert Szent-Gyorgyi Clinical Centre, University of Szeged, Szeged, Hungary
}

(Received: 3 May 2015; accepted: 15 October 2015)

We describe a rare case of Lyme disease complicated by unilateral neuroretinitis in the right eye. We report a case of a 27 -year-old woman with blurred vision on her right eye. Because of the suspicion of optic neuritis (multiplex sclerosis) neurological examination was ordered. Surprisingly, computer tomography of the brain revealed incomplete empty sella, which generally results not monocular, but bilateral optic nerve swelling. Opthalmological examination (ophthalmoscopy and optical coherence tomography) indicated not only monocular optic nerve, but retinal oedema next to the temporal part of the right optic disk. Visual evoked potentials (VEP) demonstrated no P100 latency delay and mild differences between the amplitudes of the responses of the left and right eye. Optical coherence tomography (OCT) demonstrated the swelling of the optic nerve head and oedematous retina at the temporal part of the disk. Suspicion of an inflammatory cause of visual disturbance blood tests was ordered. Doxycycline treatment was ordered till the result of the blood test arrived. The Western blot and ELISA test were positive for Borrelia burgdorferi sensu lato. Following one week corticosteroide and ceftriaxone treatments, the patient displayed a clinical improvement. Unilateral neuroretinitis with optic disk swelling due to neuroborreliosis is a rare complication and in many cases it is difficult to distinguish between inflammatory and ischemic lesions. Further difficulty in the diagnosis can occur when intracranial alterations such as empty sella is demonstrated by $\mathrm{CT}$ examination.

Keywords: Lyme disease, neuroretinitis, incomplete empty sella, visual evoked potential, optical coherence tomography

*Corresponding author; E-mail: vmelinda74@gmail.com 


\section{Introduction}

Lyme disease is a multisystem infectious disease caused by tick-borne spirochetes of the Borrelia burgdorferi sensu lato complex. Differential diagnosis and laboratory diagnosis of this infection and interpretation of the results are quite difficult. Serological testing, such as enzyme immunoassay (EIA) and Western blot can be useful, if the possibility of infection has been arisen. Cranial neuropathies are common, but neuroretinitis has been reported in a few isolated cases. The relationship between neuroretinitis and Lyme disease has not been established and the result of recent studies remains controversial [1-6]. We describe the case history of a patient with Lyme disease complicated by neuroretinitis in the right eye and incomplete empty sella.

\section{Case presentation}

Because of the confusion feeling and blurred vision in the right eye a 27-year-old woman was admitted to the Department of Neurology. Computer tomography of the brain was ordered, which revealed incomplete empty sella. Ophthalmological examination showed normal visual acuity (right eye: 1.0; left eye: 1.0) with relative central scotoma in the right side. Fundus examination showed unilateral swelling of the optic nerve head in the right eye. OCT examination revealed fluid accumulation was next to the disk in the outer nuclear layer of the retina (Figures 1 and 2a, 2b). Visual evoked potentials (VEP) reported no P100 latency delay and mild amplitude difference between the responses on the left and right eye. Pattern electroretinogram (PERG) was normal (Figure 3).

Borrelia specific antibodies, including $\operatorname{IgM}$ and IgG (Borrelia recombinant IgM, IgG ELISAs (Biomedica, Austria) were elevated in her serum sample (18.61 BBU/ml; 72.22 BBU/ml). Positive serology was confirmed using Western blot (recomLine Borrelia IgM, Mikrogen; recomLine Borrelia IgG, Mikrogen (Table I). The patient was treated with a 2 -week course of intravenous antibiotic (ceftriaxone) followed by intramuscular injections for 1 week, without corticotherapy. The intraretinal oedema significantly decreased in the papillomacular region (Figure 2c, 2d). 

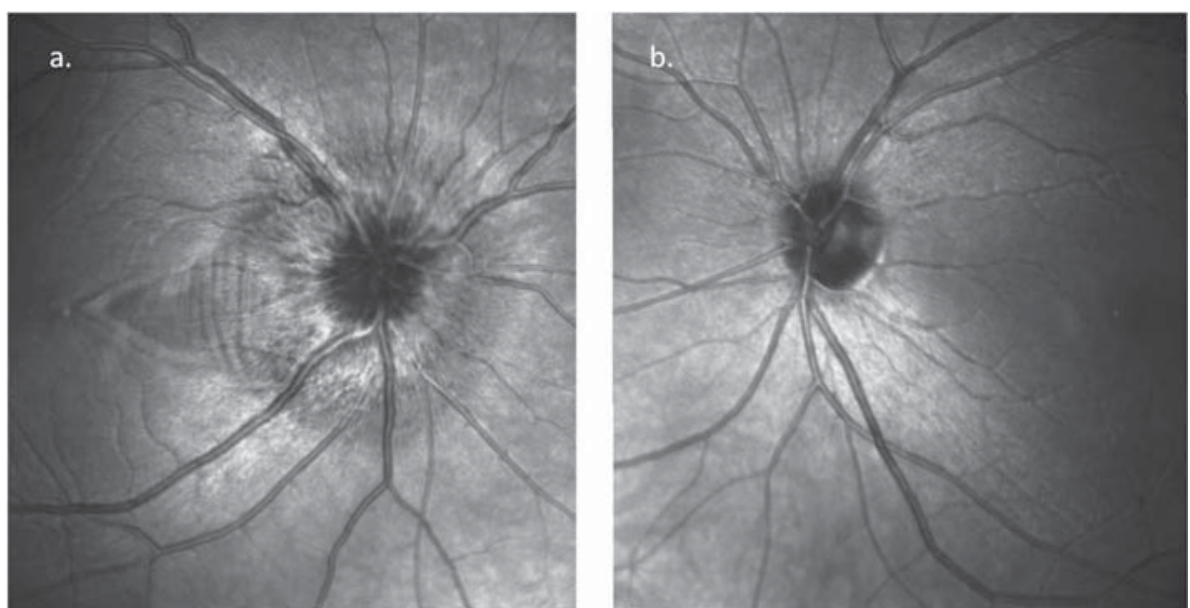

Figure 1. (a) Swelling of the optic nerve head in the right eye was detected before the ceftriaxone treatment, the papilla was normal on the left eye (b)

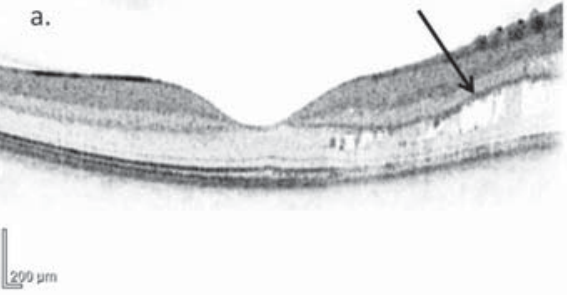

c.

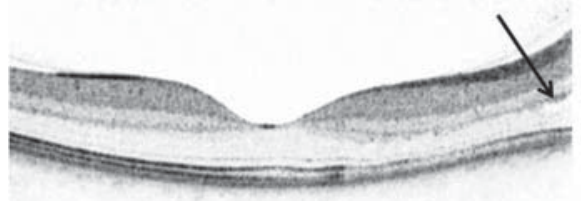

Irgosm

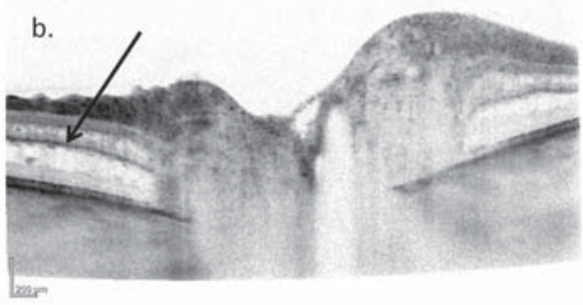

d.

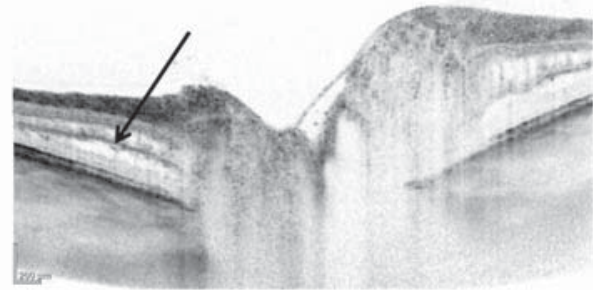

Figure 2. Optical coherence tomography (OCT) of the macular area and the optic nerve head. Before treatment, cystoid spaces were seen in the outer nuclear layer of the papillomacular area (a and $\mathrm{b}$ arrows). Four weeks after the initial examination, with systemic antibiotic and steroid treatment the cystoid oedema almost disappeared (c and d arrows), although the swelling of the optic nerve head still persisted ( $b$ and $d)$ 

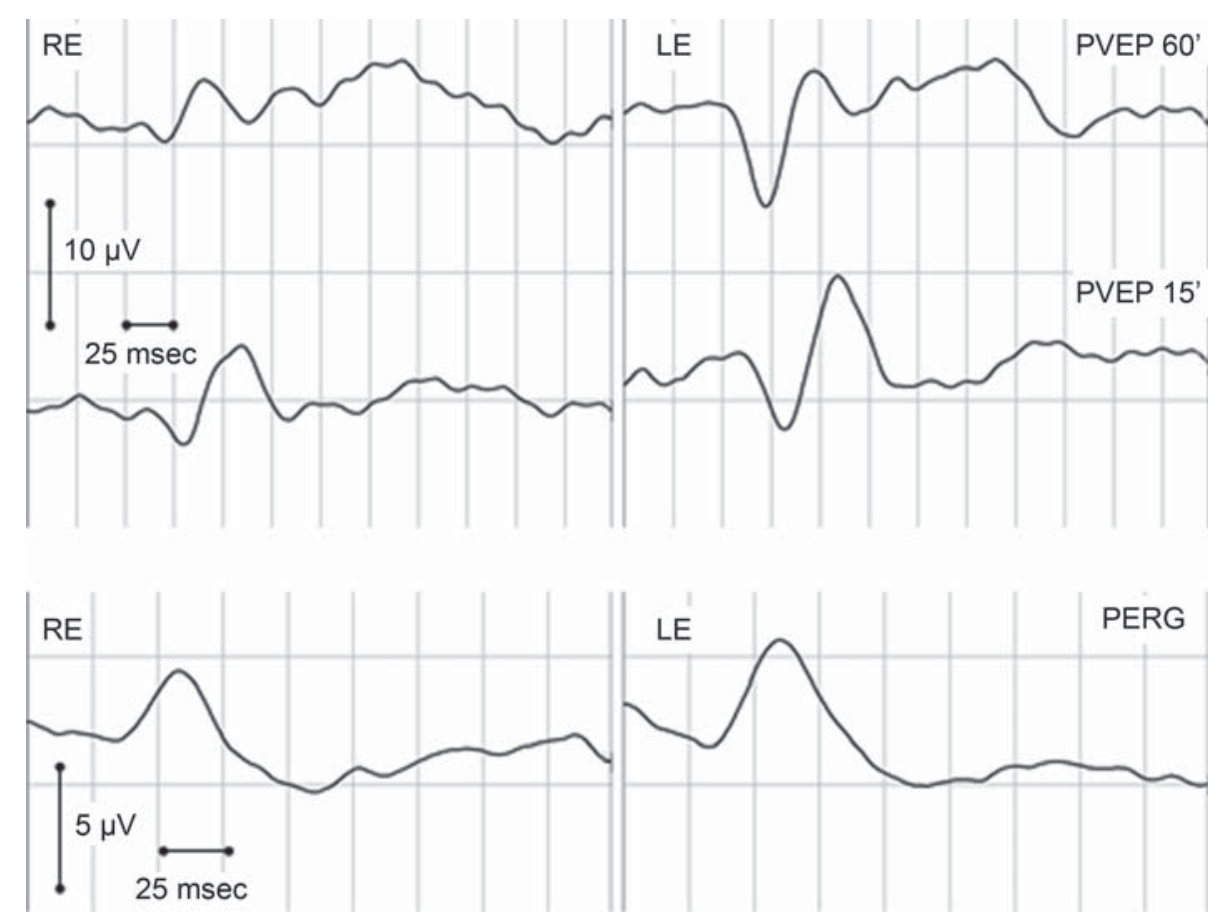

Figure 3. Visual evoked potential and pattern electroretinogram had normal implicit time.

On 60' size pattern stimulation the amplitude decreased compared to the contralateral side, while no side difference was detected in the amplitude of the responses to $15^{\prime}$ size pattern stimulation

Table I. Results of the Western blot and ELISA test

\begin{tabular}{lcc}
\hline Name of the examinations & Titres & Results of the test \\
\hline Borrelia recombinant IgM ELISA test (Biomedica) & $18.610 \mathrm{BBU} / \mathrm{ml}$ & POSITIVE \\
Borrelia recombinant IgG ELISA test (Biomedica) & $72.220 \mathrm{BBU} / \mathrm{ml}$ & POSITIVE \\
recomLine Borrelia IgM (Mikrogen) & & POSITIVE \\
recomLine Borrelia IgG (Mikrogen) & POSITIVE \\
\hline
\end{tabular}

\section{Conclusions}

Very few cases of retrobulbar optic neuritis, papillitis, neuroretinitis and ischemic optic neuropathy have shown evidence of a strong association with Lyme neuroborreliosis and there are sufficient evidence between Lyme disease and posterior uveitis [6]. Optic neuropathy in Lyme neuro-borreliosis is a typi- 
cally rare complication. However, in adult cases, special attention seems reasonable in patients with unilateral painless visual disturbance, bilateral optic nerve head swelling with or without an elevated cerebrospinal fluid opening pressure $[1,3,5,6]$. The differential diagnosis in a patient with subtle optic disc oedema and subretinal fluid includes both infectious and non-infectious entities. Noninfectious causes include optic neuritis, diabetic papillopathy, sarcoidosis, malignant hypertension, anterior ischemic optic neuropathy and retinal hemangioblastoma of the optic nerve. Infectious etiologies include Bartonella henselae, syphilis, toxoplasmosis, toxocariasis, Lyme disease and herpes viruses. In endemic areas, any optic neuropathy may still be considered for a Lyme neuroborreliosis work-up. The use of specific laboratory methods (EIA) and for confirmation Western blot and the accepted criteria for establishing the diagnosis of Lyme neuroborreliosis is emphasised.

\section{Limitation of the Case Report}

Infectious origin of unilateral neuroretinitis were scarlely investigated, only Lyme serology were presented. After the recovery of the patient it would have been feasible to make a control serology, preferably with samples collected min. 3 months apart, monitoring the reactivity. Because of the travel of patient back to Latvia, we cannot make follow up of Lyme reactivity in Hungary.

\section{Acknowledgement}

The authors express their thanks for the assistants to help the management of the patient and her samples.

\section{Conflict of Interest}

No conflict of interest.

\section{References}

1. Krim, E., Guehl, D., Burbaud, P., Lagueny, A.: Retrobulbar optic neuritis: A complication of Lyme disease? J Neurol Neurosurg Psychiatry 78, 1409-1410 (2007).

2. Sibony, P., Halperin, J., Coyle, P. K., Patel, K.: Reactive Lyme serology in optic neuritis. J Neuroophthalmol 25, 71-82 (2005). 
3. Yukawa, E., Urano, T., Nakahara, M., Miyata, K., Matsuura, T., Taketani, F., Hara, Y., Mochizuki, M.: Pattern-reversal visual evoked potentials in patients with human T-lymphotrophic virus type 1 uveitis. Curr Eye Res 31, 37-42 (2006).

4. Stanek, G., O’Connell, S., Cimmino, E., Aberer, E., Kristoferitsch, W., Granström, M., Guy, E., Gray, J.: European Union Concerted Action on risk assessment in Lyme borreliosis (EUCALB): Clinical case definitions for Lyme borreliosis. Wien Klin Wochenschr 108, 741-747 (1996).

5. Halperin, J. J., Logigian, E. L., Finkel, M. F., Pearl, R. A.: Practice parameters for the diagnosis of patients with nervous system Lyme borreliosis (Lyme disease). Neurology 46, 619-627 (1996).

6. Träisk, F., Lindquist, L.: Optic nerve involvement in Lyme disease. Curr Opin Ophthalmol 23, 485-490 (2012). 